Medical University of Silesia, Katowice: ${ }^{3}$ Department of Radiology and Radiodiagnostics, Medical University of Silesia, Zabrze, Poland

Background and Aims Neuroinfection is an entity with possible serious subsequent complications. Early and precise diagnosis can help in purposeful treatment and accurate prognosis. The aim of the study was the analysis of principles and value of neuroimaging in the diagnostic process in pediatric neuroinfection.

Methods The retrospective analysis comprised 74 patients diagnosed with encephalitis and/or meningitis. The cohort was divided into two groups: A (meningitis; $n=45$ ) and $B$ (encephalitis and meningoencephalitis, $\mathrm{n}=29$ ). Data obtained from medical records (medical history, signs and symptoms, results of laboratory tests and radiological imaging) were investigated. Computer tomography (CT) or magnetic resonance (MR) were performed in the study group.

Results In the group A first CT examination revealed abnormalities in $9.7 \%$ of patients, in the group B - in $28 \%$ of children. MR examination showed pathological brain area in $79.3 \%$ of patients in group B. High signal in SE/T2 was observed in $95.7 \%$ and in FLAIR in $86.9 \%$. In $90 \%$ of analyzed group B the disturbances in DWI were noted.

Conclusions CT examination preformed in the initial stadium of meningitis and/or encephalitis has limited diagnostic value for recognizing of inflammation. The characteristic of the abnormalities revealed by MR enables to recognize inflammation changes in central nervous system and their localization can direct diagnostic process. The presence of brain tissue alterations in MR image has significant correlation with clinical symptoms like seizures, consciousness disturbances and neurological deficits. The most sensitive sequences in estimation of the inflammation activity process are DWI and FLAIR.

\section{THIOPENTONE INFUSION IN ICU- THERE IS MORE TO THIS THAN MEETS THE EYE}

doi:10.1136/archdischild-2012-302724.0962

G Subramanian, Q Mok, J Brierley. PICU, Great Ormond Street Hospital, London, UK

Thiopentone infusion is used to manage patients with Traumatic Brain Injury with persistent raised Intracranial pressure (ICP) and in treating intractable seizures. Pupillary abnormalities (unequal or fixed and dilated) can occur as a complication. The aim of this study was to determine factors associated with this complication.

Methods Retrospective review of patients admitted to a tertiary level PICU between 2000-2011 receiving thiopentone infusion or $>2$ boluses (not as a part of Rapid sequence intubation). 59 patients were identified. Patients who had dilating eye drops were excluded. Quantitative variables are expressed as mean and Standard deviation, qualitative variables as percentages. Student's t-test is used for comparison of quantitative variables.

Results The mean age of study population was 6.5years $( \pm 5.3)$. Thiopentone was used for ICP management in 44 (74.6\%) and seizure control in 15 (25.4\%) patients. 13 (22\%) episodes of pupillary abnormality (of which 8 had fixed and dilated pupils) were recorded in $12(20.3 \%)$ patients. Dose $/ \mathrm{kg}(\mathrm{p}=0.675)$, maximum rate of infusion $(p=0.37)$ or duration of infusion $(p=0.51)$ were not significantly related to the occurrence of this complication. Age $>9$ years was significantly associated $(\mathrm{p}=0.05$ ) with abnormal pupillary reaction.

Conclusion High dose, maximum infusion rate or longer duration of thiopentone infusion were not associated with abnormal pupillary reaction. Drug levels were not done in all and it is not known whether this complication is a result of genotypic variation in drug metabolism. It is however important to have continuous EEG monitoring to ensure that the optimal dose is administered to the patient.

\section{PREGNANCY-ASSOCIATED PLASMA PROTEIN A LEVELS AND NEONATAL COMPLICATIONS IN POST-TERM PREGNANCIES}

doi:10.1136/archdischild-2012-302724.0963

${ }^{1}$ AR Zizzo, ${ }^{2}$ Kirkegaard, ${ }^{3}$ TB Henriksen, ${ }^{2} \mathrm{~N}$ Uldbjerg. ${ }^{1}$ Perinatal Epidemiology Research Unit; '2Department of Obstetrics and Gynecology; ${ }^{3}$ Department of Pediatrics, Aarhus University Hospital, Skejby, Aarhus, Denmark

Objectives To assess the association between serum pregnancyassociated plasma protein A (PAPP-A) measured in the first trimester and perinatal complications in post-term pregnancies.

Methods A total of 4948 singleton pregnant women, who delivered after $40^{+0}$ gestational weeks, were included at Aarhus University Hospital between January 2005 and December 2007. Serum levels of PAPP-A were determined at the first-trimester screening for Down syndrome $\left(\right.$ GA $\left.8^{+0}-13^{+6}\right)$. Labor was not induced routinely until $42^{+0}$ weeks of gestation. However, in women with diabetes mellitus, gestational diabetes, preeclampsia, hypertension, prelabor rupture of membranes, twin pregnancies, fetal growth restriction and intrahepatic cholestasis, labor were induced by 40 completed weeks or earlier and were not included in the study.

Results In post-term pregnancies first-trimester serum PAPP-A below $0.4 \mathrm{MoM}$ was associated with small weight for gestation $\left(<5^{\text {th }}\right.$ centile $)\left(\mathrm{OR}_{\text {adj }} 1.7,95 \% \mathrm{CI} 0.9-3.0\right)$, Apgar score of less than 7 at 5 minutes ( $\mathrm{OR}_{\text {adj }} 5.4,95 \% \mathrm{CI} 2.0-14.3$ ), admission to the neonatal intensive care unit $\left(\mathrm{OR}_{\text {adj }} 1.5,95 \%\right.$ CI 1.0-2.3), and newborn hypoglycemia $\left(\mathrm{OR}_{\text {adj }} 3.4,95 \% \mathrm{CI} 1.8-6.4\right)$. In neonates with small weight for gestation the risk of hypoglycemia was increased considerably (OR 14.6, 95\% CI 3.4-58.0).

Conclusions Low first trimester serum PAPP-A was associated with increased neonatal morbidity in post-term pregnancies, particularly in newborns with low birth weight. Thus, PAPP-A may qualify the timing of induction of labor in these pregnancies and low PAPP-A may indicate more closely follow-up when past 40 weeks of gestation.

\section{IS THE PLACEBO EFFECT REVEALABLE IN NEWBORNS? RESULTS FROM AN RCT IN OSTEOPATHY}

doi:10.1136/archdischild-2012-302724.0964

${ }^{1} \mathrm{~F}$ Cerritelli, ${ }^{2} \mathrm{G}$ Barlafante, ${ }^{2} \mathrm{C}$ Renzetti, ${ }^{2} \mathrm{~F}$ Ricci, ${ }^{2} \mathrm{~N}$ Loprete, ${ }^{1} \mathrm{G}$ Pizzolorusso, ${ }^{3} \mathrm{P}$ Fusilli, ${ }^{3} \mathrm{C}$ D'Incecco. 'European Institute for Evidence Based Osteopathic Medicine; ${ }^{2}$ Accademia Italiana Osteopatia Tradizionale; ${ }^{3}$ Neonatal Intensive Care Unit, Pescara Hospital, Pescara, Italy

Background and Aims Placebo effect has been largely studied and debated in medicine. Interestingly the majority of studies focused on children and adults but not on newborns. In the field of osteopathic medicine, few studies documented this effect using sham therapy. A previous study showed the association between OMT and LOS on newborns. However, no research were conducted on the placebo effect on newborns osteopathically treated. Therefore the aim of this study is to detect the association between placebo treatment and change in clinical outcome in newborns.

Methods A double blinded randomized control trial was carried out on 250 preterm newborns, GA $>29$ and $<37 \mathrm{w}$ and free of medical complications. After enrollment, all subjects were randomly assigned to study $(\mathrm{N}=107)$ and control group $(\mathrm{N}=143)$. All preterms received routine pediatric care and osteopathic sham therapy was administered to the study group only for the entire period of hospitalization. Primary outcome was to evaluate the effectiveness of sham therapy in reducing LOS.

Results At entry, univariate statistical analysis showed no differences between groups. At the end of the study, after adjusting for all potential confounders, generalized linear model analysis showed no difference on the primary outcome (mean difference between study and control group: 2.444 ; $95 \%$ CI $-0.447,5.337$; $\mathrm{p}=0.09$ ). 